\title{
Stainability and Fine Structure of Intracytoplasmic Inclusion Bodies in the Locus Ceruleus of Mouse
}

\author{
Yoshimitsu Y. Katoh ${ }^{1 \#}$, Kazuyoshi Sakai ${ }^{1}$, Chiyuki Kaneko ${ }^{2}$, Masato Abe $^{2}$ \\ ${ }^{1}$ Department of Anatomy, School of Health Sciences, Fujita Health University, Toyoake, Aichi, Japan \\ ${ }^{2}$ Department of Pathology, School of Health Sciences, Fujita Health University, Toyoake, Aichi, Japan \\ Email: " ykatoh@fujita-hu.ac.jp
}

Received September 28, 2013; revised October 29, 2013; accepted November 8, 2013

Copyright (C) 2013 Yoshimitsu Y. Katoh et al. This is an open access article distributed under the Creative Commons Attribution License, which permits unrestricted use, distribution, and reproduction in any medium, provided the original work is properly cited.

\begin{abstract}
The present study compared intracytoplasmic inclusion bodies in the locus ceruleus (LC) of mouse with protein bodies in the LC of human. Phosphotungstic acid-hematoxylin (PTAH) and the Mallory method were used as the anionic stains. The inclusion bodies and protein bodies, which stained with PTAH and the Mallory method in the brain, contain proteins that appear to belong to the same family. Although both inclusion and protein bodies were formed with the same arginine composition, their distribution in the brain was similar and similar physiological changes by morbidity were observed, and the fine structure of the inclusion bodies and protein bodies appeared to be different. The present findings suggest that mouse inclusion bodies and human protein bodies are different.
\end{abstract}

Keywords: Inclusion Body; Protein Body; Locus Ceruleus (LC); Mouse; Human

\section{Introduction}

In humans and non-human primates, the locus ceruleus (LC) corresponds to the region in the dorsolateral portion of the rostral mesencephalic and caudal mesencephalic tegmentum [1]. In the mouse, this nucleus is localized to the lateral part of the periventricular gray matter and is composed of closely packed, medium-sized nerve cells [2]. The LC contains noradrenaline neurons that project widely throughout the central nervous system [3]. When we stained paraffin sections from mouse LC with the Holmes silver impregnation method, we noticed small, silver-positive structures [4]. Furthermore, using hematoxylin-eosin staining, we also observed faintly eosinophilic small bodies in the cytoplasm [4]. The existence of nucleolus-like inclusion bodies has been demonstrated in nerve cells of the central nervous system in normal mice and rats using electron microscopy (EM) [5,6]. These inclusion bodies are devoid of a limiting membrane and comprise aggregates of granular and/or filamentous materials. It has been reported that intracytoplasmic inclusion bodies contain protein and some RNA [4,7-9]. Hol-

\footnotetext{
*This work was supported in part by a grant-in-aid from Fujita Health University, Japan.

${ }^{\#}$ Corresponding author.
}

mes's silver-positive bodies were identified in neuronal cytoplasm with silver staining of paraffin sections. The nucleolus-like inclusion bodies were demonstrated using EM $[4,10]$. We proved that the silver-positive bodies identified using the Holmes method and the inclusion bodies demonstrated with EM were the same. We subsequently modified the Holmes method to shorten the reaction time for Holmes silver staining [11,12].

It is well known that primates have melanin granules in the perikarya and dendrites of neurons in the LC in the brain [13]. Furthermore, neurons in the LC contain spherical protein bodies (acidophilic bodies) containing proteins and/or peptides, which are distributed in the soma and dendrites of human monoamine neurons [14-16].

The present study aimed to compare mouse intracytoplasmic inclusion bodies with human spherical protein bodies.

\section{Materials and Methods}

Seven normal ddY male mice (body weight, about $20 \mathrm{~g}$ ) were used for this experiment. All animals were deeply anesthetized with ether then perfused with fixative solution. The protocol for the use of these animals was approved by the Animal Care and Use Committee of Fujita Health University. 


\subsection{LM Methods}

Seven mice were perfused with $2 \%$ paraformaldehyde and $2.5 \%$ glutaraldehyde in $0.1 \mathrm{M}$ phosphoric acid buffer ( $\mathrm{pH}$ 7.4) fixative solution via the left ventricle. The five mouse brains were removed and post-fixed in the same fixative solution for three days. After postfixation the brain was dehydrated with ethanol and embedded in paraffin.

Each set of sections from the mouse was stained with the modified Holmes method [11], which improved Holmes's original silver method [12]. The modified Holmes method is a simple and easy method for staining inclusion bodies, and the staining quality is similar to the original method. Using the modified Holmes method, we searched for inclusion bodies in the cytoplasm of LC nerve cells. A second set of sections from the mouse brain, adjacent to the previous ones, was stained with phosphortungstic acid hematoxylin (PTAH) after acidified potassium permanganate oxidation, as previously described [14]. A third set of sections from the mouse brain, adjacent to the previous two sets, was stained with Mallory's modified trichrome method, as previously described [16].

\subsection{EM Methods}

For electron microscopic observation of normal sections, the two mouse brains were placed in a fixative similar to that used in the above experiment for one hour. The mouse brains were cut with a vibratome into $150 \mu \mathrm{m}$ thick sections. The sections were used as normal EM material following standard EM protocols. The sections were fixed in 2\% osmium tetroxide and embedded in Epon. In addition, $1 \mu \mathrm{m}$ thick sections were observed under the light microscope with toluidine blue staining. The LC region was identified, trimmed, and ultrathin sections were cut and stained with uranyl acetate and lead hydroxide before observation under the electron microscope.

\section{Results}

\subsection{Light Microscopic Observation}

When paraffin sections of mouse LC were stained using the modified Holmes method, nucleoli and chromatin of nerve cell nuclei, microfilaments in the cytoplasm, nerve axons, and nuclei of glial and endothelial cells showed strong positive staining, and the nucleoplasm of nerve cells showed medium-grade staining. Cytoplasm showed no staining. However, the positive-staining body (1.0 $3.0 \mu \mathrm{m}$ in diameter) was recognized in the cytoplasm of most LC neurons with the modified Holmes method (Figure 1).

In both PTAH and Mallory's trichrome-stained preparations of mouse LC, except for the neural nucleolus, the erythrocytes, the glial and endothelial nuclei and the myelin sheaths, little else seemed to stain in the tissue. How- ever, closer observation of the cytoplasm revealed that an inclusion body was often encountered in the cytoplasm of the mouse LC neurons. The inclusion bodies were spherical and ranged in size from visible to $1.0-3.0 \mu \mathrm{m}$ in diameter (Figures 2 and 3). In paraffin sections of mouse LC, the inclusion bodies were stained blue with PTAH (Figure 2), and they were stained red by Mallory's trichrome method (Figure 3). Nucleoli, erythrocytes and myelin sheaths were also intensely stained by each method. The inclusion bodies of mouse showed the same stainability as acidophilic granules.

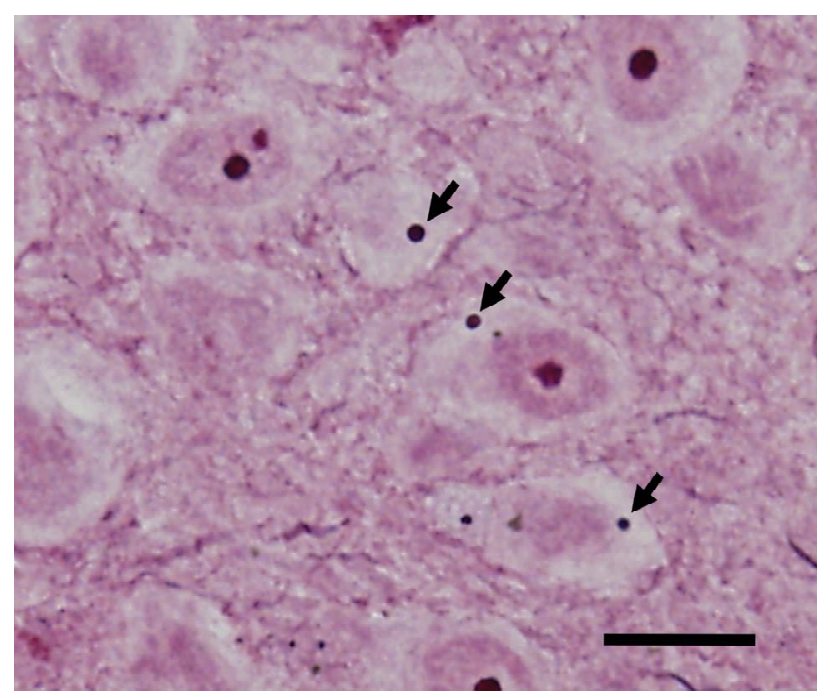

Figure 1. Light micrograph of a paraffin-embedded frontal section (thickness $5 \mu \mathrm{m}$ ) of the mouse LC stained using the modified Holmes method. Neurons of the mouse LC usually have intracytoplasmic inclusion bodies (arrows). Scale bar $=20 \mu \mathrm{m}$.

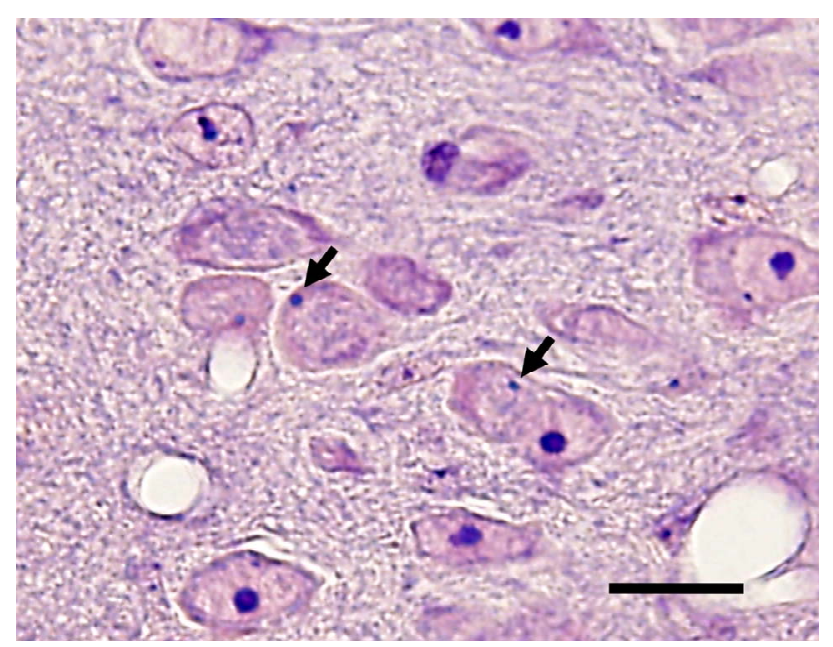

Figure 2. Light micrograph of a paraffin section of the mouse LC in the frontal plane. A $5 \mu \mathrm{m}$ thick paraffin section is stained with PTAH after acidified potassium permanganate oxidation. Inclusion bodies are stained blue dots (arrows). Bar $=20 \mu \mathrm{m}$. 


\subsection{Electron Microscopic Observation}

In thin sections of the mouse LC prepared using standard EM methods, we noted small, round, electron-dense bodies in the cytoplasm of neurons in the LC. The fine structure lacked a limiting membrane, and indicated a small accumulation of electron-dense balls (Figure 4). The shape, size and distribution in the neuronal cytoplasm corresponded to acidophilic granules. The inclusion bodies were $1.0-3.0 \mu \mathrm{m}$ in diameter. Though the inclusion bodies often contact with cell organelles such as free ribosomes, rough endoplasmic reticulum (rER),

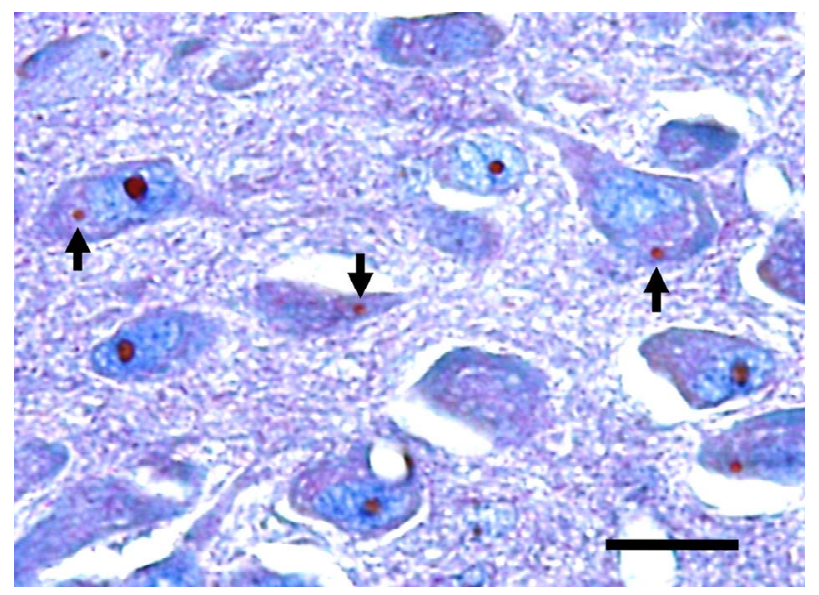

Figure 3. Paraffin section stained with the Mallory's modified trichrome method. Inclusion bodies are stained red like nucleoli (arrows). Bar $=20 \mu \mathrm{m}$.

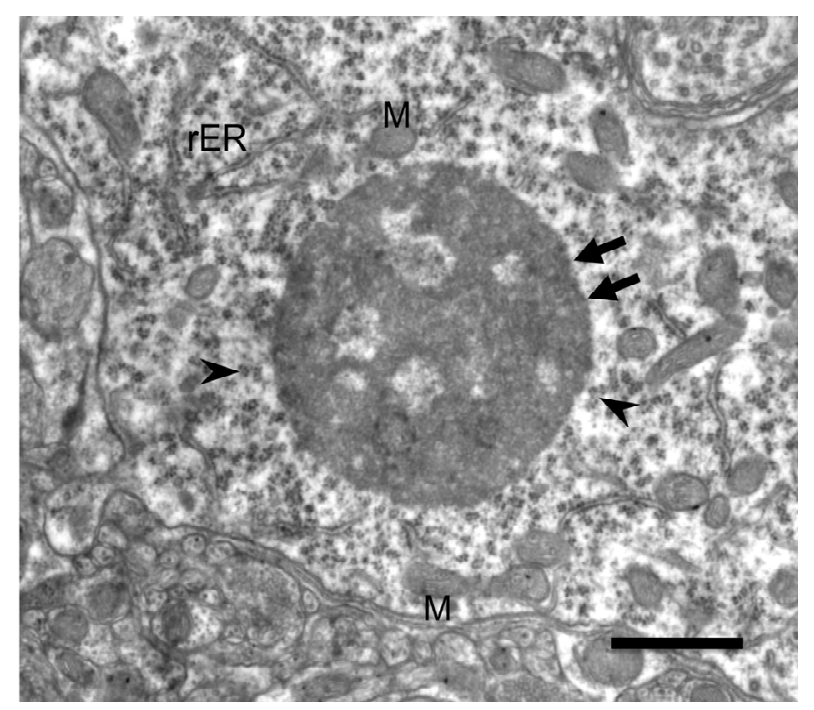

Figure 4. Electron micrograph of the inclusion body in the cytoplasm of locus ceruleus cells. No limiting membrane (arrows) is observed. The inclusion body consists of an aggregate of medium-density granules, with frequent clear spaces of varying shapes and sizes. The inclusion body is surrounded by free ribosomes (arrowheads), rough endoplasmic reticulum (rER), and mitochondria $(\mathrm{M})$. Bar $=1$ $\mu \mathrm{m}$. and mitochondria, their relation to cytoplasmic organellae was unclear.

\section{Discussion}

In the present study, the PTAH method and the Mallory method were used. The PTAH stain has a high affinity for basic amino acids (lysine, arginine, histidine) [17,18]. The PTAH staining of the nucleolus and protein bodies reflects the high concentrations of arginine in those structures [19-21]. The inclusion bodies of mouse (observed in this study) and protein bodies of human [16] have identical histochemical reactions in brain tissue, where they are easily detected by acid fuchsine in the Mallory method as well as by the PTAH method. Although the presence of acidophilic components in the inclusion and protein bodies has been suggested by previous experiments $[4,14-16]$, the nature of these molecules remains unclear.

From our studies, the inclusion bodies appeared to be globes of $1.0-3.0 \mu \mathrm{m}$ in diameter. The fine structure lacked a limiting membrane, and indicated a small accumulation of electron-dense balls. Inclusion bodies were reportedly formed from rER and free ribosomes in cultured mouse cells that include an insertion of the Huntington's disease gene, using in situ hybridization-electron microscopy [22]. We also thought the inclusion body to be composed of the product of the rER and free ribosomes from our EM observations of hamster LC [23]. The protein bodies from the human were composed of the dense homogeneous substances and a dense rim and a limiting double membrane [26]. They stand out and are differentiated from all other neuronal inclusions, especially melanin. In earlier EM observations, spherical dense bodies that originated inside mitochondria were found in the LC of Japanese macaque monkeys [24] and humans $[25,26]$. The findings of all previous studies have consistently reported that the dense homogeneous substance of protein bodies is stored gradually in the matrix of the mitochondria and finally a round homogeneous mass remains and is surrounded by the two mitochondrial membranes [27]. Therefore, the fine structure of the inclusion bodies and protein bodies was quite different.

We reported that the preferential occurrence of inclusion bodies in mouse seems to be in the LC, some hypothalamic nuclei and other parts of the autonomic and limbic systems as well as in the circumventricular structures [28]. Consistent with our observations, it was reported that the protein bodies exist in the aminergic cell groups $[15,16]$.

Our previous studies used experimental conditions such as dehydration/fasting or reserpine administration for mice, and the numbers of inclusion bodies decreased [29]. It was reported that the protein bodies were found to be severely depleted or missing from the neurons of 
substantia nigra (SN) and LC in Parkinsonian brains [15] and disrupted in the LC in depression [30]. These findings point to the importance of the metabolic economy of catecholamine neurons since protein bodies in the SN and LC were lost in parallel with the dopamine loss in the $\mathrm{SN}$, with the reduced concentrations of norepinephrine in the $\mathrm{LC}$ reported in Parkinsonian brains [31,32] and with disturbed metabolism of norepinephrine in cerebrospinal fluid in depression [33].

\section{Conclusion}

Based on the present findings, we can conclude that although both inclusion bodies and protein bodies are formed with the same composition (arginine), their place of origin and fine structure were different. In addition, the numbers of both the inclusion bodies and protein bodies decrease in various disease states. The argininerich basic protein of the spherical bodies, it is argued that, may be involved in the modulation of excitability of the catecholamine neurons in the rodent and the human. To better understand the functional significance of the protein bodies and inclusion bodies, further study will be necessary.

\section{REFERENCES}

[1] G. V. Russel, "The Nucleus Locus Coeruleus (Dorsolateralis Tegmenti)," Texas Reports on Biology \& Medicine, Vol. 13, 1955, pp. 939-988.

[2] Y. Katoh, M. Kanematsu and N. Shimizu, "Light-Microscopic Studies of Locus Coeruleus Region of the Mouse," Brain and Nerve, Vol. 36, 1984, pp. 185-191.

[3] D. G. Amaral and H. M. Sinnamon, "The Locus Coeruleus. Neurobiology of a Central Noradrenergic Nucleus," Progress in Neurobiology, Vol. 9, 1977, pp. 147-196. http://dx.doi.org/10.1016/0301-0082(77)90016-8

[4] Y. Katoh and N. Shimizu, "The Light and Electron-Microscopic Localization of Intracytoplasmic NucleolusLike Bodies in the Mouse Brain Stained by Holmes' Silver Method," Archivum Histologicum Japonicum, Vol. 45, 1982, pp. 325- 333.

[5] I. Kawabata, "Electron Microscopy of the Rat Hypothalamic Neurosecretory System. Il. Nucleolus-Like Inclusion Bodies in the Cytoplasm of Neurosecretory Cells," Archivum Histologicum Japonicum, Vol. 26, 1965, pp. 101-113.

[6] A. P. Anzil, H. Herrlinger and K. Blinzinger, "Nucleolus Inclusions in Neuronal Perikarya and Processes: Phase and Electron Microscope Observations on the Hypothalamus of the Mouse," Zeitschrift für Zellforschung und Mikroskopische Anatomie, Vol. 146, No. 3, 1973, pp. 329-337. http://dx.doi.org/10.1007/BF02346225

[7] M. A. Grillo, "Cytoplasmic Inclusions Resembling Nucleoli in Sympathetic neurons of Adult Rats," The Journal of Cell Biology, Vol. 45, No. 1, 1970, pp. 100-117. http://dx.doi.org/10.1083/jcb.45.1.100
[8] R. C. Santolaya, "Nucleolus-Like Bodies in the Neuronal Cytoplasm of the Mouse Arcuate Nucleus," Zeitschrift für Zellforschung und Mikroskopische Anatomie, Vol. 146, No. 3, 1973, pp. 319-328. http://dx.doi.org/10.1007/BF02346224

[9] C. Hindelang-Gertner, M. E. Stoeckel, A. Porte, H. D. Dellmann and B. Madarász, "Nematosomes or NucleolusLike Bodies in Hypothalamic Neurons, the Subfornical Organ and Adenohypophysial Cells of the Rat," Cell and Tissue Research, Vol. 155, No. 2, 1974, pp. 211-219. http://dx.doi.org/10.1007/BF00221355

[10] Y. Katoh, "The Nucleolus-Like Bodies in the Mouse Locus Coeruleus," Brain and Nerve, Vol. 34, 1982, pp. 39-45.

[11] Y. Katoh and N. Shimizu, "Identity of Holmes Positive Bodies with Electron Microscopically Demonstrable Nucleolus-Like Bodies in Neuronal Cytoplasm," Stain Technology, Vol. 57, 1982, pp. 83-89.

[12] W. Holmes, "Silver Staining of Nerve Axons in Paraffin Sections," The Anatomical Record, Vol. 86, No. 2, 1943, pp. 157-187. http://dx.doi.org/10.1002/ar.1090860205

[13] M. Bazelon, G. M. Fenichel and J. Randa II, "Studies on Neuromelanin. I. A Melanin System in the Human Adult Brain Stem," Neurology, Vol. 17, 1967, pp. 512-519. http://dx.doi.org/10.1212/WNL.17.5.512

[14] M. R. Issidorides, C. Mytilineou, W. O. Whetsel1 Jr. and M. D. Yahr, "Protein-Rich Cytoplasmic Bodies of Substantia Nigra and Locus Coeruleus. A Comparative Study in Parkinsonian and Normal Brain," Archives of Neurology, Vol. 35, 1978, pp. 633-637. http://dx.doi.org/10.1001/archneur.1978.00500340009002

[15] M. R. Issidorides and M. T. Panayotacopoulou, "Histochemical Protein-Markers of Monoamine Neurons," Neuroscience Letters, Vol. 1, 1978, p. 270.

[16] M. T. Panayotacopoulou and M. R. Issidorides, "Histochemical Protein-Markers of Monoamine Cell Bodies in Man," Archives of Neurology, Vol. 39, 1982, pp. 635-639. http://dx.doi.org/10.1001/archneur.1982.00510220033007

[17] J. Y. Terner, "Phosphotungstic Acid-Hematoxylin: Reactivity in Vitro," Journal of Histochemistry \& Cytochemistry, Vol. 14, No. 4, 1966, pp. 345-351. http://dx.doi.org/10.1177/14.4.345

[18] D. M. Phillips and P. Simson, "Identification of Some Peptides from an Arginine-Rich Histone and Their Bearing on the Structure of Deoxyribonucleohistone," Biochemical Journal, Vol. 82, 1962, pp. 236-241.

[19] B. E. Magun and J. W. Kelly, "A New Fluorescent Method with Phenanthrenequinone for the Histochernical Demonstration of Arginine Residues in Tissues," Journal of Histochemistry \& Cytochemistry, Vol. 17, No. 12, 1969, pp. 821-827. http://dx.doi.org/10.1177/17.12.821

[20] G. Quintarelli, M. Bellocci and R. Geremia, "On Phosphotungstic Acid Staining IV. Selectivity of the Staining Reaction," Journal of Histochemistry \& Cytochemistry, Vol. 21, No. 2, 1973, pp. 155-160. http://dx.doi.org/10.1177/21.2.155

[21] M. R. Issidorides and G. D. Pappas, "Fine Structure of Neuronal Spherical Arginine-Rich Bodies of Substantia 
Nigra and Locus Coeruleus in the Human Brain," Human Neurobiology, Vol. 6, 1988, pp. 239-246.

[22] A. Yanai, R. Fujinaga, J. Kawano and K. Shinoda, "In Situ Hybridization for Electron Microscopy; with Special Reference to the Stigmoid Body," Anatomical Science International, Vol. 79, 2004, p. 383.

[23] Y. Y. Katoh, E. Yamazaki, K. Taniguti, K. Yamada and G. Isomura, "Light and Electron Microscopic Observation of Intracytoplasmic Inclusion Bodies in the Locus Coeruleus of the Hamster," Archives of Histology and Cytology, Vol. 69, No. 2, 2006, pp. 129-134. http://dx.doi.org/10.1679/aohc.69.129

[24] K. Hirosawa, "Electron Microscopic Studies on Pigment Granules in the Substantia Nigra and Locus Coeruleus of the Japanese Monkey (Macaca Fuscata Yakui)," Zeitschrift für Zellforschung und Mikroskopische Anatomie, Vol. 88, No. 2, 1968, pp. 187-203. http://dx.doi.org/10.1007/BF00703907

[25] L. S. Forno and R. L. Norville, "Ultrastructural Study of the Human Locus Coeruleus (in Middle-Aged and Older Persons with and without Parkinsonism)," Proceedings of the VII International Congress on Neuropathology, Excerpta Medica and Akademia Lmiado, Amsterdam, 1975, pp. 459-462.

[26] S. Sekiya, M. Tanaka, S. Hayashi and S. Oyanagi, "Lightand Electron-Microscopic Studies of Intracytoplasmic Acidophilic Granules in the Human Locus Coeruleus and Substantia Nigra," Acta Neuropathologica, Vol. 56, No. 1, 1982, pp. 78-80. http://dx.doi.org/10.1007/BF00691186

[27] H. H. Goebel, "Dense Core Mitochondria in Peripheral Ganglia of Man,” Acta Neuropathologica, Vol. 36, 1976, pp. 85-90. http://dx.doi.org/10.1007/BF00685151

[28] Y. Y. Katoh, C. Kishi, H. Takano, K. Yamada and G. Isomura, "Localization of Nucleolus-Like Inclusion Bodies in the Neuronal Cytoplasm in Mouse Brains: A Study Using Holmes Modified Method," Structure and Function, Vol. 1, 2003, pp. 3-11.

[29] Y. Katoh and N. Shimizu, "Quantitative Changes of Holmes Positive Nucleolus-Like Inclusion Bodies in the Mouse Locus Coeruleus under Various Experimental Conditions," Archivum Histologicum Japonicum, Vol. 46, No. 4, 1983, pp. 491-500. http://dx.doi.org/10.1679/aohc.46.491

[30] M. R. Issidorides, "Cellular Factors in Manic Depressive Illness: Blood and Brain," In: C. N. Stefanis, Ed., Recent Advances in Depression, Pergamon Press, London, 1983, pp. 99-107.

[31] H. Ehringer and O. Hornykiewicz, "Beurteilung von Noradrenalin und Dopamin (3-hydroxytyramin) im Gehirn des Menschen und ihr Verhalten bei Erkrankungen des Extrapyramidalen Systems," Klinische Wochenschrift, Vol. 38, 1960, pp. 1236-1239. http://dx.doi.org/10.1007/BF01485901

[32] O. Hornykiewicz and S. J. Kish, "Biochemical Pathophysiology of Parkinson's Disease," Advances in Neurology, Vol. 45, 1987, pp. 19-34.

[33] R. M. Post, E. K. Gordon, F. K. Goodwin and W. E. Bunney Jr., "Central Norepinephrine Metabolism in Affective Illness: MHPG in the Cerebrospinal Fluid," Science, Vol. 179, 1973, pp. 1002-1003.

http://dx.doi.org/10.1126/science.179.4077.1002 